\title{
The properties of a large volume-limited sample of face-on low surface brightness disk galaxies
}

\author{
G.-H. Zhong ${ }^{1,2,3,4}$, Y.-C. Liang ${ }^{1,2}$, F.-S. Liu ${ }^{5}$, F. Hammer ${ }^{4}$, K. Disseau ${ }^{4,6}$ and L.-C. Deng ${ }^{1,2}$ \\ 1 National Astronomical Observatories, Chinese Academy of Sciences, Beijing 100012, China; \\ ghzhong@nao.cas.cn \\ 2 Key Laboratory of Optical Astronomy, National Astronomical Observatories, Chinese Academy of \\ Sciences, Beijing 100012, China \\ 3 Graduate University of Chinese Academy of Sciences, Beijing 100049, China \\ ${ }^{4}$ GEPI, Observatoire de Paris-Meudon, 92195 Meudon, France \\ 5 College of Physical Science and Technology, Shenyang Normal University, Shenyang 110034, China \\ 6 Univ Paris Diderot, Sorbonne Paris Cit, GEPI, UMR8111 CNRS, Observatoire de Paris, F-92190 \\ Meudon, France
}

\begin{abstract}
We select a large volume-limited sample of low surface brightness galaxies (LSBGs, 2,021) to investigate their statistical properties and their differences from high surface brightness galaxies (HSBGs, 3,639) in details. The distributions of stellar masses of LSBGs and HSBGs are nearly the same and they have the same median values. Thus this volume-limited sample have good completeness and further remove the effect of stellar masses on their other properties when we compare LSBGs and HSBGs. We found that LSBGs tend to have lower stellar metallicities, and lower effect dust attenuations indicating that they have lower dust, than HSBGs. The LSBGs have relatively higher stellar mass-to-light ratios, higher gas fraction, lower star forming rates (SFRs), and lower specific SFRs than HSBGs. Moreover, with the decreasing surface brightness, gas fraction increase, while the SFRs and specific SFRs decrease rapidly for the sample galaxies. This could mean that the star formation histories between LSBGs and HSBGs are different, HSBGs may have stronger star forming activities than LSBGs.
\end{abstract}

Key words: galaxies: fundamental parameters, galaxies: general, galaxies: statistics, galaxies: stellar content

\section{INTRODUCTION}

Galaxies with surface brightness fainter than $\mu_{0}(B)=21.65 \pm 0.3$ mag $\operatorname{arcsec}^{-2}$ are well known as low surface brightness galaxies (LSBGs, Freeman 1970). Yet, owing to their faintness compared with the night sky, they are hard to find. Hence their contribution to the local galaxy population has been underestimated for a long time. However it is found that LSBGs could represent a significant fraction of galaxy number density in the universe (O'Neil \& Bothun 2000; Minchin et al. 2004; Trachternach et al. 2006) and may comprise up to half of the local galaxy population (McGaugh et al. 1995).

During the last four decades, LSBGs are studied widely both in observations (Impey et al. 1996; O'Neil et al. 1997; Trachternach et al. 2006; Haberzettl et al. 2007a; Pizzella et al. 2008; Ulmer et al. 2011; Morelli et al. 2012) and theoretical work (Dalcanton et al. 1997; Alard 2011). Especially, thanks to the modern digital sky surveys, a wealth of observational data, with high quality, is undoubtedly important to allow us to study the photometric and/or spectroscopic properties of LSBGs with large 
samples and even in multi-wavelengths. For example, (1) the statistical properties (Zhong et al. 2008, 2010), the metallicities (Liang et al. 2010), the environment (Rosenbaum et al. 2004, 2009; Galaz et al. 2011), and the stellar red holes (Bergvall et al. 2010) of LSBGs from Sloan Digital Sky Survey (SDSS); (2) the stellar populations (Zhong et al. 2008) and HI observation (Monnier Ragaigne et al. 2003a, 2003b) of LSBGs from Two Micron All Sky Survey (2MASS); (3) Star Formation Efficiency (Boissier et al. 2008) from Galaxy Evolution Explorer (GALEX); (4) infrared properties (Hinz et al. 2007) from Spitzer Space Telescope. In addition, the multi-wavelength SEDs are also investigated (Gao et al. 2010).

From the studies above, LSBGs, generally, are found to have lower metallicities (McGaugh 1994; Galaz et al. 2006; Haberzettl et al. 2007b; Liang et al. 2010), lower surface densities that could explain their slow evolution (Mo et al. 1994; Gerritsen \& de Blok 1999; van den Hoek et al. 2000), lower fraction of AGN (Impey et al. 2001; Mei et al. 2009; Liang et al. 2010; Galaz et al. 2011), lower star formation rate (SFRs, van den Hoek et al. 2000), higher $M_{*} / L$ ratio (Sprayberry et al. 1995), higher gas fraction, and they are located in lower densities regions (Mo et al. 1994; Rosenbaum et al. 2004, 2009; Galaz et al. 2011) than what is typically found in HSBGs.

Despite these impressive progresses, there are still several challenges about LSBGs, such as many aspects of their formation and evolution. Moreover, the studies of $M_{*} / L$, SFRs or gas contents of LSBGs are based on very small samples, less than two hundred LSBGs (e.g. McGaugh \& de Blok 1997; Burkholder et al. 2001). In particular, a magnitude-limited survey will be affected by selection effects, which result from the inability of the survey to detect fainter galaxies at larger redshifts (e.g. Impey et al. 1996, Zhong et al. 2008). One way to avoid these effects is through using a volume-limited sample, in which a maximum redshift and minimum absolute magnitude are chosen so that a complete sample is obtained in this redshift and magnitude ranges. Some properties could be different between the volumelimited sample and the magnitude-limited sample. For example, in Zhong et al. (2008), the relation between disk scale-length and $\mu_{0}(B)$ are different between total sample and the volume-limited sample. This difference is caused by the selection of volume-limited sample from the magnitude-limited sample. When selecting volume-limited LSBGs at $z<0.1$, we exclude two parts: on the one hand, LSBGs with relatively lower redshift (smaller distance) and lower luminosity (see bottom-left of Fig. 11) are excluded, which should have smaller disk scale-length (see Fig. 4 and Fig. 5 of Zhong et al. 2008) as well as fainter surface brightness (see Fig. 3 of Zhong et al. 2008); on the other hand, LSBGs with relatively higher redshift (larger distance) and higher luminosity (see top-right of Fig. 11 are excluded, which should have larger disk scale-length (see Fig. 4 and Fig. 5 of Zhong et al. 2008) as well as brighter surface brightness (see Fig. 3 of Zhong et al. 2008). Excluding LSBGs with smaller disk scale-length at fainter surface brightness end and larger disk scale-length at the brighter surface brightness end from Fig. 7 of Zhong et al. (2008) results in the relation between disk scale-length and surface brightness of their Fig $13 \mathrm{~g}$, the more obvious correlation between $\log h$ and $\mu_{0}(B)$. Furthermore, in Galaz et al. (2011), they point out that the strong dependence of the absolute magnitude versus redshift in the SDSS spectroscopic catalog (a magnitude-limited catalog), and the trend of the absolute magnitude on the galaxy size (see Fig. 1 of Galaz et al. 2011 for more details).

In this work, with the advent of the large sky survey of the SDSS, it is now possible to dramatically extend the studies of SFRs, $M_{*} / L$, and gas contents of LSBGs in sample size. Moreover, this large amount of high quality data will be undoubtedly important to allow us to study the properties of those galaxies more carefully. Furthermore, in order to avoid the bias of magnitude-limited samples, we constrain on a large volume-limited sample of LSBGs (more than two thousands) to study their statistical properties in detail, and then compare them with HSBGs. The volume-limited samples could be a fair comparison between LSBGs and HSBGs, and they improve the completeness of the samples.

This is one of our series work (Zhong et al. 2008, 2010; Liang et al. 2010; Gao et al. 2010; Chen et al. in preparation) to study the properties of a large sample of LSBGs and compare their differences with HSBGs. This paper is organized as follows. We describe our sample selections in Sect. 2 and present the results in Sect. 3. In Sect. 4 we discuss our results. In Sect. 5 we summarize this work. Throughout the paper, a cosmological model with $H_{0}=70 \mathrm{~km} \mathrm{~s}^{-1} \mathrm{Mpc}^{-1}, \Omega_{M}=0.3$ and $\Omega_{\lambda}=0.7$ is adopted. 


\section{SAMPLES}

The data analyzed in this study are drawn from the SDSS, which is an imaging and spectroscopic survey that will obtain photometry of one-quarter of the sky and spectra of nearly one million objects. The imaging data were collected in $u, g, r, i, z$ band mounted on the SDSS $2.5 \mathrm{~m}$ telescope at Apache Point Observatory. The spectra are flux- and wavelength-calibrated, with 4,096 pixels from 3,800 to $9,200 \AA$ at $R \sim 1,800$. The details of the survey strategy and overview of the data pipelines and products can be found in York et al. (2000) and Stoughton et al. (2002). In this paper, for the sample galaxies, the stellar masses, mass-to-light ratios, and effective dust attenuations are from Kauffmann et al. (2003); the SFRs and specific SFRs are from Brinchmann et al. (2004); the stellar metallicities are from Gallazzi et al. (2005). All of those data can be found in the MPA/JHU websit@ 1 .

The same as Zhong et al. (2008), the sample is selected from the main galaxy sample (MGS, Strauss et al. 2002) of SDSS Data Release Foun (DR4, Adelman-McCarthy et al. 2006). We prefer to use the DR4 because some of the parameters we use here have just been updated to DR4 by the MPA/JHU groups, e.g. stellar metallicities from Gallazzi et al. (2005). The detailed criteria of sample selection can be found in Zhong et al. (2008). We describe it briefly as follows.

1. fracDev $<00.25$, the parameter fracDev $v_{r}$ indicates the fraction of luminosity contributed by the de Vaucouleurs profile relative to exponential profile in the $r$-band. This is to select disk dominated galaxies which can minimize the effect of bulge light on the disk galaxies.

2. $b / a>0.75$ (corresponding to the inclination $i<41.41$ degree), this is to select nearly face-on galaxies, which can minimize the extinction. $a$ and $b$ are the semi-major and semi-minor axes of the fitted exponential disk respectively.

3. $M_{B}<-18$, this is to exclude a few dwarf galaxies contained in the sample.

4. We select objects with $\mu_{0}(B) \geq 22.0 \mathrm{mag} \operatorname{arcsec}^{-2}$ as LSBGs and $\mu_{0}(B)<22.0 \mathrm{mag} \mathrm{arcsec}^{-2}$ as HSBGs. The $\mu_{0}(B)$ are calculated following the method of Zhong et al. (2008, their Eq. 6). After this step, we get 12,282 LSBGs and 18,051 HSBGs.

5. In order to avoid the bias introduced from the differences in both the redshift and absolute magnitude distributions for the samples, we extract volume-limited samples from the $M_{r}-z$ plane by considering $z<0.1$ and galaxies brighter than the corresponding $M_{r}$. After this step, we get 3,313 LSBGs and 4,722 HSBGs.

The relationship between $r$ band absolute magnitude and redshift for LSBGs is showed in Fig. 1 The vertical line refers to $z=0.1$, while the horizontal line is the corresponding $M_{r}$. The galaxies located in the top-left region within the solid lines are selected as our volume-limited sample.

6. In this work, in order to obtain the values of the property parameters, we just select galaxies with all measurements of stellar masses, mass-to-light ratios, effective dust attenuations, SFRs and specific SFRs, and stellar metallicities. At last, we get the volume-limited samples of 2,021 LSBGs and 3,639 HSBGs following the volume-limited sample selections of Zhong et al. (2008).

All the magnitudes, we quote above, are $K$-corrected and Galactic reddening corrected (Blanton et al. 2005).

\section{RESULTS}

Using large volume-limited samples of LSBGs and HSBGs, we present the properties of LSBGs (e.g. stellar metallicities, effective dust attenuations, mass-to-light ratios, and SFRs) and compare them with HSBGs. The stellar metallicities are from Gallazzi et al. (2005), in which they determine the stellar metallicities by a spectroscopic method that used a few line strength indices to measure the stellar population properties of galaxies. The stellar masses, mass-to-light ratios, and effective dust attenuations in $z$ band are from Kauffmann et al. (2003), all of which are generated by using a large library of Monte

\footnotetext{
${ }^{1}$ http://www.mpa-garching.mpg.de/SDSS/DR4

2 http://www.sdss.org/DR4
} 


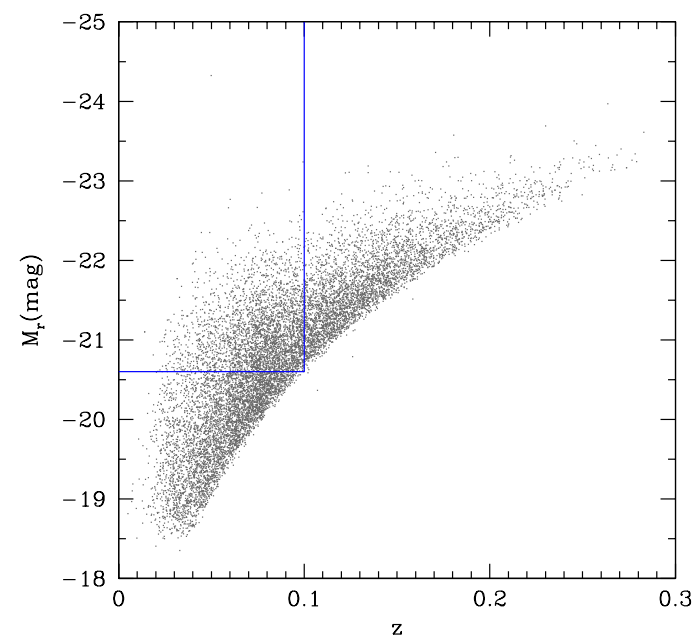

Fig. 1 The relationship between $r$ band absolute magnitude and redshift for LSBGs. The vertical line refers $z=0.1$ and the horizontal line is the corresponding $M_{r}$. The top-left region with solid lines is selected as volume-limited sample.

Carlo realizations of different star formation histories, including starbursts of varying strength and a range of metallicities. The SFRs are from Brinchmann et al. (2004), who build up a picture of the nature of star-forming galaxies at $z<0.2$ by comparing physical information extracted from the emission lines (e.g. $\mathrm{H} \alpha$ ) with continuum properties, and develop a method for aperture correction using resolved imaging. This method takes out essentially all aperture bias in their SFRs estimates, allowing an accurate estimates of the total SFRs in galaxies. One may wish to refer to the papers of Gallazzi et al. (2005), Kauffmann et al. (2003), and Brinchmann et al. (2004) about the properties of galaxies in SDSS.

\subsection{Distributions of stellar masses, stellar metallicities, effective dust attenuations and stellar mass-to-light ratios}

Fig. 2] shows the histograms distributions of some parameters for LSBGs and HSBGs. They are the distributions of the stellar masses (Fig. 23), the stellar metallicities (Fig. 2b), effective dust attenuations in $z$ band (Fig. 2r), and dust-corrected mass-to-light ratios in $z$ band (Fig. 21 d), respectively. The shadowed regions are for LSBGs, while the white regions are for HSBGs.

From the distributions of stellar masses in Fig. 2a, we can see that LSBGs and HSBGs span nearly the same range and are with very similar distributions. Kolmogorov-Smirnov tests show that the different mean of distributions between LSBGs and HSBGs is only 6.6\%. The median values of stellar masses of volume-limited samples of LSBGs and HSBGs are both $2.19 \times 10^{10} M_{\odot}$. This result in our volumelimited samples is different from Liang et al. (2010) who showed that galaxies with lower surface brightness have smaller stellar masses generally. This difference may be because they use magnitude-limited samples that could be affected by the completeness, while we use volume-limited samples to avoid such selection effects. Moreover, the nearly same stellar masses distributions between LSBGs and HSBGs can help to get a more fair comparison in the following sections since the affecting of stellar masses is nearly removed.

The stellar metallicities $\left[\log \left(Z / Z_{\odot}\right)\right]$ of LSBGs also span nearly the same range as that of HSBGs (Fig. 2b), however, the median value of stellar metallicities for LSBGs is -1.95 which is 0.11 dex (1.3 times) lower than that of HSBGs (-1.84). Kolmogorov-Smirnov tests show that the different mean of 

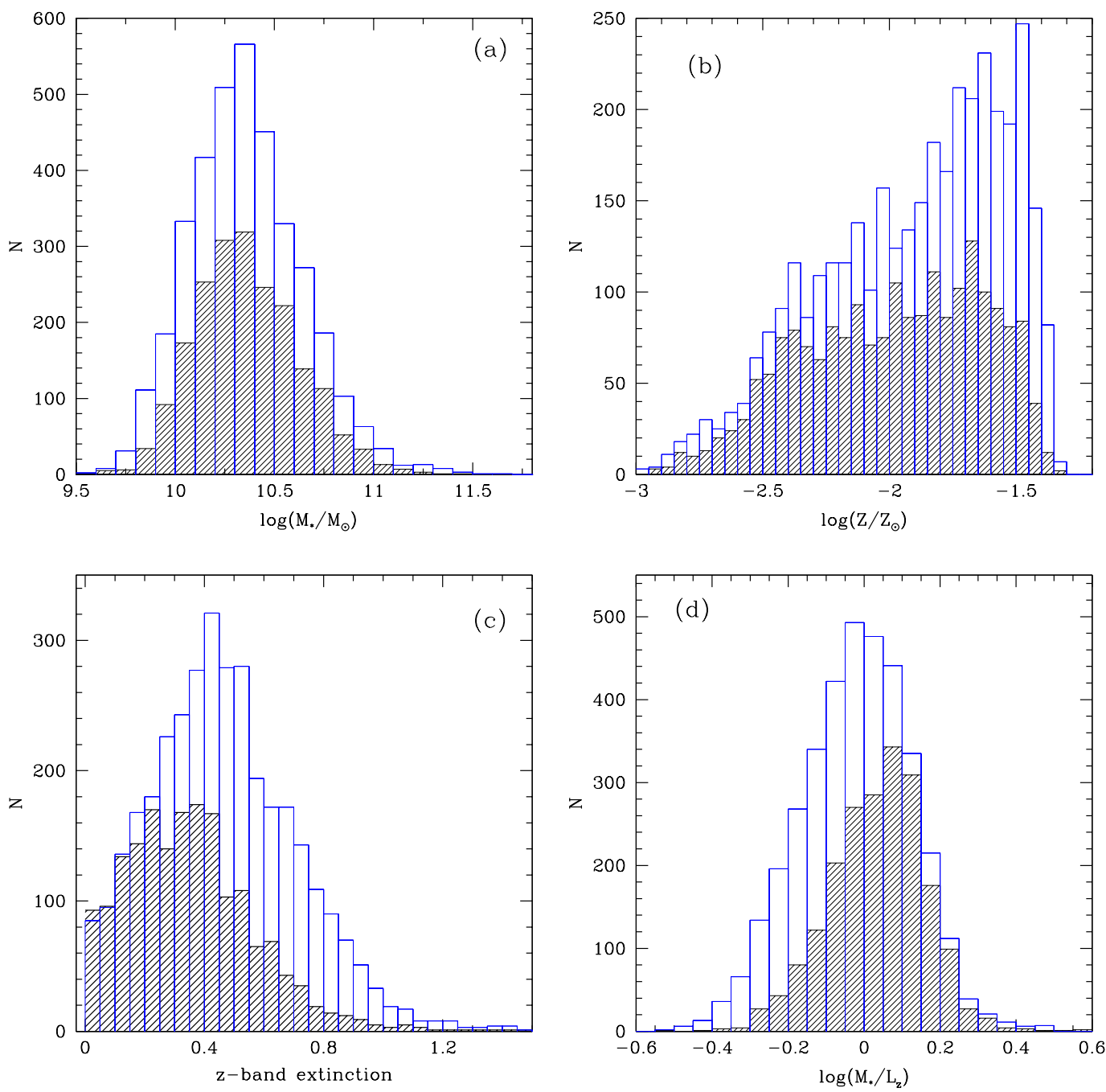

Fig. 2 Histograms distributions of some parameters for LSBGs (shadowed regions) and HSBGs (white regions): (a). the distributions of stellar masses $\left(\log \left(M_{*} / M_{\odot}\right)\right)$, (b). the stellar metallicities $\left(\log \left(Z / Z_{\odot}\right)\right)$, and (c). the effective dust attenuations in $z$ band, (d) the histogram distributions of dust-corrected mass-to-light ratios in $z$ band.

distributions between LSBGs and HSBGs is $16.0 \%$. The result that LSBGs have lower stellar metallicities is consistent with the lower metallicities of LSBGs found in gas-phase (McGaugh 1994, Galaz et al. 2006, Haberzettl et al. 2007b, Liang et al. 2010). Liang et al. (2010) found that the decreasing of metallicities with decreasing surface brightness could be due to the decreasing stellar masses with decreasing surface brightness. In our volume-limited samples, however, LSBGs have the similar stellar masses distributions with HSBGs, yet they still have lower metallicities. Thus, lower stellar masses is not enough to explain the lower metallicities of LSBGs. The LSBGs could have different star formation histories from HSBGs.

Fig. 2r shows that the LSBGs have lower effective dust attenuations in $z$ band,. The median value is $0.34 \mathrm{mag}$, which is $0.11 \mathrm{mag}$ smaller than that of HSBGs whose median value of effective dust 

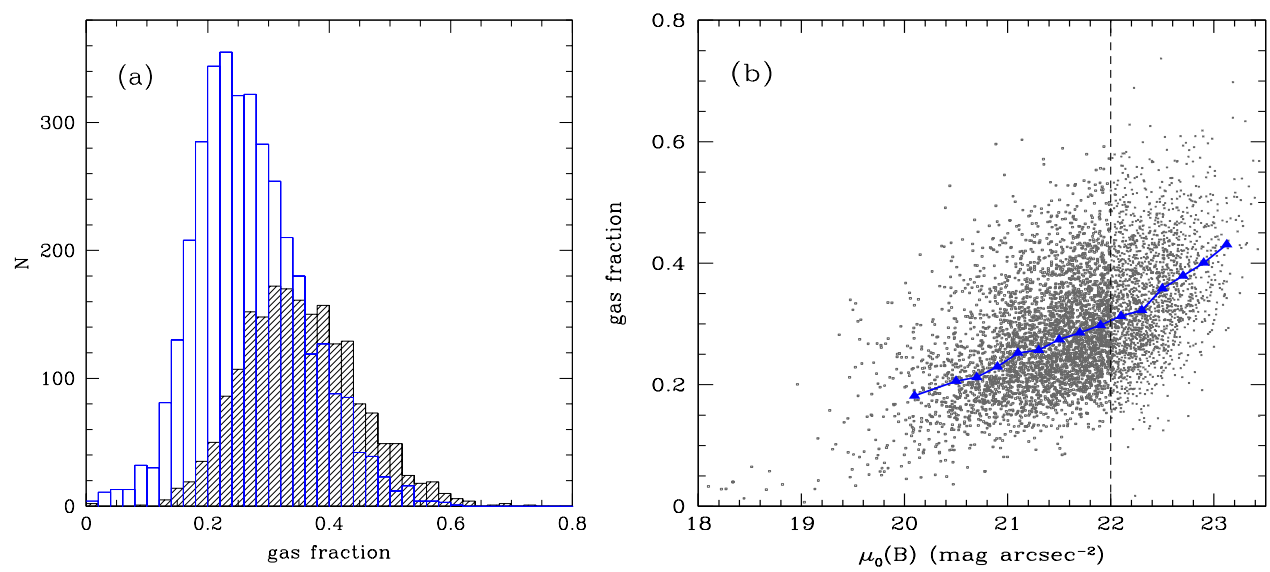

Fig. 3 The histogram distributions of gas fraction (a), shadowed and white regions are for LSBGs and HSBGs respectively. The relation between gas fraction and surface brightness (b). The solid triangles and line denote the median values with surface brightness bins. The vertical dashed line marks the range of LSBGs and HSBGs.

attenuations in $z$ band is 0.45 . Kolmogorov-Smirnov tests show that the different mean of distributions between LSBGs and HSBGs is up to $26.4 \%$. The lower effective dust attenuations in red band ( $z$ band) is consistent with the results in $V$ band (e.g. Liang et al. 2010), which means that LSBGs could contain less dust than HSBGs (McGaugh 1994; Hinz et al. 2007).

In Fig. 2d, we show the distributions of dust-corrected $z$ band mass-to-light ratios, shadowed and white regions are for LSBGs and HSBGs respectively. The LSBGs tend to have higher mass-to-light ratios than HSBGs. Kolmogorov-Smirnov tests show that the different mean of distributions between LSBGs and HSBGs is $23.7 \%$. The median value of stellar mass to $z$ band luminosity $\operatorname{ratios}\left(M_{*} / L_{z}\right)$ is 1.11 that is 0.15 (1.4 times) higher than that of HSBGs with median $M_{*} / L_{z}$ of 0.96 . The high $M_{*} / L_{z}$ ratios either point toward galaxies early in their evolution that have not converted gas into stars or reflect more recent accumulation of gas by an evolved stellar population (Burkholder et al. 2001). This result may also indicate different star formation histories between LSBGs and HSBGs.

\subsection{Gas contents}

Gas fraction can be used to quantify galaxy evolution (e.g. McGaugh \& de Blok 1997; Burkholder et al. 2001). Assuming a constant $M_{*} / L$, it is said that LSBGs are considered as gas-rich galaxies, which may result from the fact that LSBGs have not converted gas into stars effectively. In our work, we first calculate the HI gas-to-stellar mass ratio $(\log [G / S])$ by using the formula of Zhang et al. (2009, see their Eq. 4 for more details)

$$
\log (G / S)=-1.73238(g-r)+0.215182 \mu_{i}-4.08451
$$

where $G$ and $S$ refer to HI mass $\left(M_{H I}\right)$ and stellar mass $\left(M_{*}\right)$, and $g, r$ are the apparent magnitudes from SDSS in $g, r$ band, both of which are $K$-corrected and Galactic reddening corrected (Blanton et al. 2005). The $\mu_{i}$ is surface brightness in SDSS $i$ band, which is defined as $\mu_{i}=m_{i}+2.5 \log \left(2 \pi R_{50}^{2}\right)$, where $m_{i}$ is the apparent magnitude in $i$-band that is also $K$-corrected and Galactic reddening corrected (Blanton et al. 2005), and $R_{50}$ is the radius (in units of arcsec) enclosing 50 per cent of the total Petrosian $i$-band flux. Then the HI mass can be calculated from $\log (G / S)$ because the stellar mass is available (Kauffmann et al. 2003). At last, one can get the gas fraction from $f_{g}=M_{g} /\left(M_{g}+M_{*}\right)$, where $M_{g}=$ $1.4 M_{H I}$ (e.g. McGaugh \& de Blok 1997; Schombert et al. 2001). 
We show the histogram of the calculated gas fraction in Fig. 3a, from which we can see that the gas fraction of LSBGs span nearly the same range as that of HSBGs but more likely to distribute in the higher gas fraction region. We try our best to find related work on gas fraction of LSBGs to compare with our results, and found three much related ones (i.e. McGaugh \& de Blok 1997; Schombert et al. 2001; Burkholder et al. 2001). The range of gas fraction of our volume-limited LSBGs is approximate from $15 \%$ to $70 \%$, which is very similar to the results in McGaugh \& de Blok (1997) for small sample of LSBGs (17\% to 77\%). Schombert et al. (2001) found that a majority of the galaxies in their disk samples have gas fraction below $50 \%$ peaked at $30 \%$, whereas in the LSB dwarf galaxies over $90 \%$ of the galaxies have gas fraction greater than $30 \%$. For our volume-limited LSBGs, most have gas fraction below $70 \%$ with a median value of $34.6 \%$. This result is higher than that of the disk sample of Schombert et al. (2001), but lower than that of their LSB dwarf galaxies. The reason is that our volume-limited LSBGs contain more brighter galaxies without any dwarf galaxies (Zhong et al. 2008). The median value of gas fraction of our LSBGs is quite similar to that of McGaugh \& de Blok (1997) with small sample of LSBGs (median value of $40.1 \%$ ), only 5.5\% higher than ours (34.6\%). Moreover, the median value of gas fraction for the our LSBGs is $8.7 \%$ higher than that of HSBGs (median value of $25.9 \%$ ), thus, LSBGs are indeed gas-rich galaxies. Kolmogorov-Smirnov tests show that the different mean of distributions between LSBGs and HSBGs is bigger, up to $48.7 \%$.

Furthermore, there is a tight correlation between gas fraction and surface brightness (the Spearman rank order correlation coefficient is 0.51 ) that galaxies with lower surface brightness have higher gas fraction (Fig. 3b). This relation has been found in some previous studies with very small samples. For example, McGaugh \& de Blok (1997) found the correlation coefficient between gas fraction and surface brightness is 0.63, which is a little tighter than ours. Schombert et al. (2001) used chemical and spectrophotometric models from Boissier \& Prantzos (2000) also predict the general trend of higher gas fraction with fainter surface brightness.

Though the correlation we find shows more scatter than those found by McGaugh \& de Blok (1997), Schombert et al. (2001), and Burkholder et al. (2001), our conclusion is similar to theirs: LSBGs tend to be gas-rich. The high gas fraction of LSBGs could indicate that either these galaxies have experienced delays in formation and are just beginning to form stars or their ongoing star formation is inefficient and/or sporadic (Burkholder et al. 2001).

Moreover, the slope of gas fraction vs. surface brightness for LSBGs is a little steeper than that of HSBGs.

\subsection{Star formation rates}

It is showed that the typical gas surface densities for LSBGs are below the Kennicutt criterion for ongoing star formation (Kennicutt 1989; van der Hulst et al. 1993) which results in a suppressed current SFRs (Bossier et al. 2008; Schombert et al. 2011). Thus the SFRs of LSBGs should be lower than that

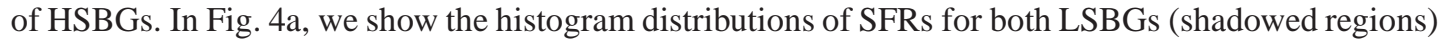
and HSBGs (white regions). We can see that LSBGs are more likely to have lower SFRs. KolmogorovSmirnov tests show that the different mean of distributions between LSBGs and HSBGs is $30.7 \%$. The median value of SFRs for LSBGs is $1.77 M_{\odot} \mathrm{yr}^{-1}$ which is $0.86 M_{\odot} \mathrm{yr}^{-1}$ smaller than that of HSBGs that have median SFRs of $2.63 M_{\odot} \mathrm{yr}^{-1}$. Moreover, the SFRs drop quickly with the decreasing surface brightness (see Fig. 4b), the Spearman rank order correlation coefficient is -0.32 , which could suggest that higher surface brightness galaxies are undergoing stronger star forming activities than the lower ones.

However the SFRs of LSBGs span a wide range, and the SFRs of LSBGs are not as low as the results in the previous studies (e.g. 0.02-0.8 $M_{\odot} \mathrm{yr}^{-1}$, van den Hoek 2000). The reason of the relatively higher SFRs for LSBGs may be that we use volume-limited sample of LSBGs, which select the brighter LSBGs. 

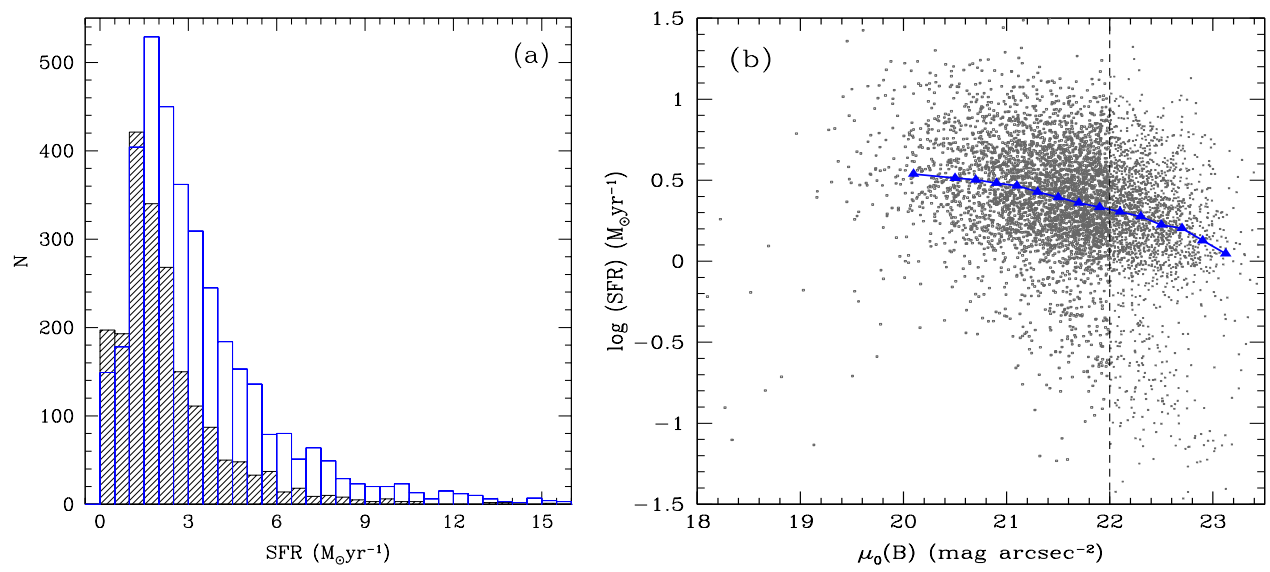

Fig. 4 The histogram distributions of SFRs (a), shadowed and white regions are for LSBGs and HSBGs respectively. The relation between SFRs and surface brightness (b). The open squares, crosses, solid triangles, solid line, and dashed line are the same as Fig. 3 .
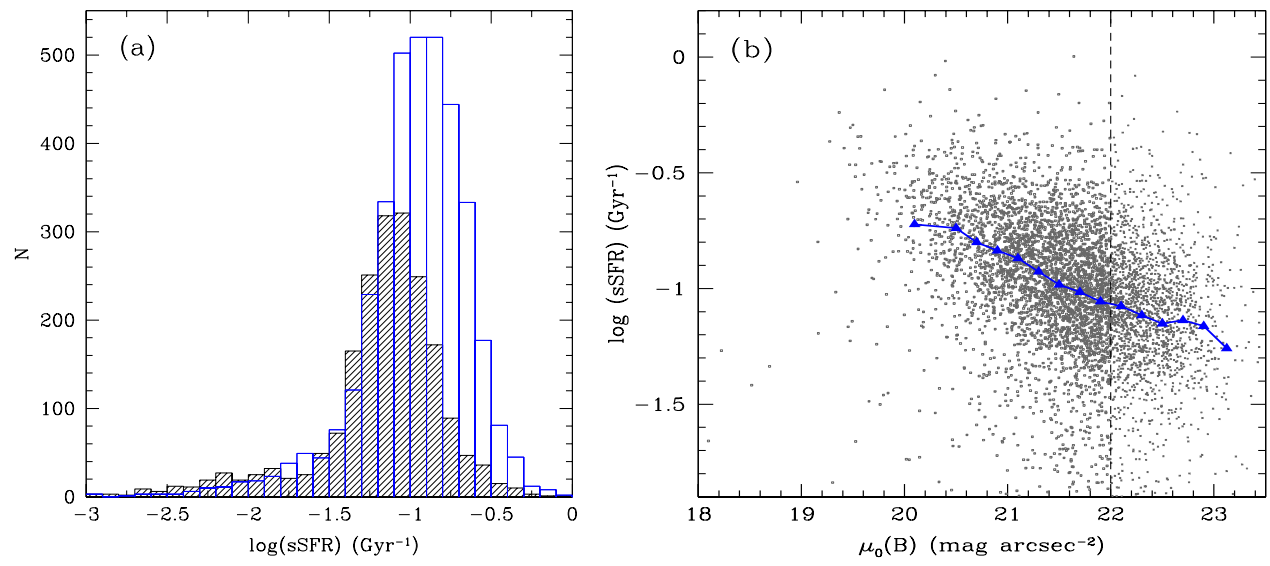

Fig. 5 The histogram distributions of specific SFRs (a), shadowed and white regions are for LSBGs and HSBGs respectively. The relation between specific SFRs and surface brightness (b). The open squares, crosses, solid triangles, solid line, and dashed line are the same as Fig. 3.

\subsection{Specific SFRs}

Given the strong correlation between SFRs and stellar masses, it is clear that by normalizing the SFRs by the stellar masses, one can more easily study the relationship between star formation activity and the physical parameters of the galaxies (Brinchmann et al. 2004). Although the distributions of stellar masses for LSBGs and HSBGs in the volume-limited samples are similar, we could further remove the effect of stellar masses by comparing the specific SFRs of LSBGs with that of HSBGs.

The specific SFRs are also from Brinchmann et al. (2004). The median value of specific SFRs for LSBGs (shadowed regions) is $-1.12 \mathrm{Gyr}^{-1}$ (Fig. 5a) which is $0.18 \mathrm{Gyr}^{-1}$ smaller than that of 


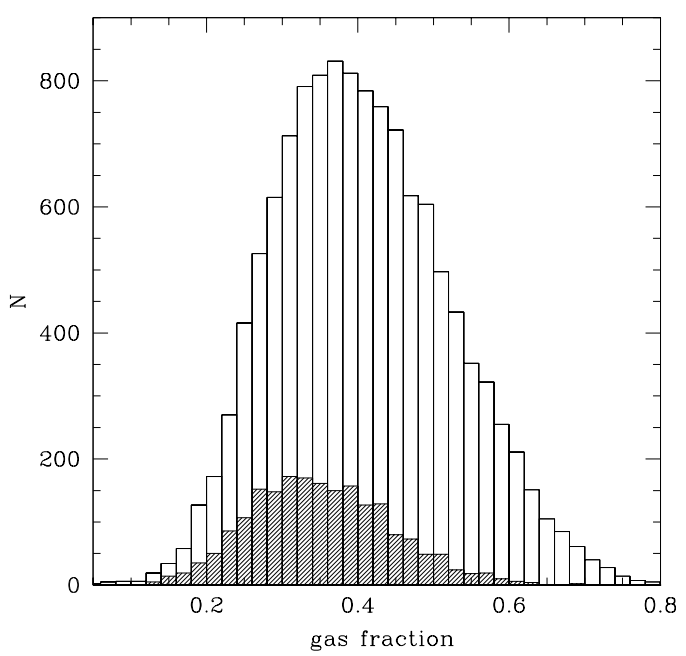

Fig. 6 The histogram distributions of gas fraction for volume-limited LSBGs $(2,021$, shadowed regions) and all LSBGs (12,282, white regions).

HSBGs (white regions). Kolmogorov-Smirnov tests show that the different mean of distributions between LSBGs and HSBGs is up to 34.9\%. This result is consistence with the result of Sect 3.3, which shows that the LSBGs have smaller SFRs than HSBGs but nearly the same stellar masses as HSBGs. Hence LSBGs should have lower specific SFRs than that of HSBGs. Furthermore, the specific SFRs decrease rapidly with the decreasing of surface brightness, i.e. the lower the surface brightness are, the lower specific SFRs they have (Fig. 5b). LSBGs with lower specific SFRs could be also related to Kennicutt criterion for ongoing star formation, and LSBGs form stars in longer periods of time (e.g. Galaz et al. 2011). The slope of specific SFRs vs. surface brightness is a little steeper than the slope of SFRs vs. surface brightness, the Spearman rank order correlation coefficient is -0.40 . This may be due to the effects of stellar masses that have been further removed in calculating specific SFRs. Both Fig. 4 and Fig. 5 show that the current star forming activities of HSBGs are more active than that of LSBGs.

The specific SFRs has often been rephrased in terms of the present to past-average star formation rate, which immediately gives an indication of the past star formation history of the galaxy and its relation to present-day activity, e.g. the birthrate parameter $b$ (the present-to-past average SFR ratio, Kennicutt et al. 1994; Brinchmann et al. 2004; Galaz et al. 2011). Following the Eq. 2 of Galaz et al. (2011), we found that there is a fraction of $88.2 \%$ for LSBGs with $b$ less than 1 , while the corresponding fraction of HSBGs is $66.8 \%$. It means that the average $b$ parameter is higher in HSBGs than in LSBGs (Galaz et al. 2011), and the average value of $b$ parameter for our LSBGs is similar to that of Galaz et al. (2011), 0.59 vs. 0.65 .

\section{DISCUSSIONS}

The previous studies about stellar metallicities, $M_{*} / L$, SFRs and gas fractions of LSBGs have been traditionally carried out with very small samples. With the advent of the large sky survey of the SDSS, it is now possible to dramatically extend these studies in size, and it may also improve the qualities because their large amount of high-quality data will be undoubtedly important to allow to study the properties of those galaxies more carefully.

Moreover nearly all the previous studies of stellar metallicities, $M_{*} / L$, SFRs and gas fractions for LSBGs are based on magnitude-limited sample, which could be affected by selection effects. We try 
to avoid the selection effects on the studies of LSBGs by using volume-limited samples in this work. And we extend much more the number of LSBGs, which help well to study the statistical properties of LSBGs. Fortunately, our volume-limited samples of LSBGs and HSBGs have very similar stellar masses that can help to get a more fair comparison between their other properties because it nearly removes the effect of stellar masses.

However, could the different properties between LSBGs and HSBGs studied above be caused by the fact that the volume-limited sample contains a larger fraction of gas-poor galaxies? Thus, we calculate the gas fraction for all the parent LSBGs (i.e 12,282 LSBGs) and HSBGs (i.e. 18,051 HSBGs) using the same formula of Zhang et al. (2009). In Fig. 6 as an example, we show the histogram distributions of gas fraction for volume-limited LSBGs (2,021, shadowed region) and all parent LSBGs (12,282, white region). The median value of gas fraction for all parent LSBGs is $39.8 \%$, which is only $5.2 \%$ higher than that of volume-limited LSBGs (34.6\%). And the median value of gas fraction for all HSBGs is $29.0 \%$ that is only $3.1 \%$ higher than that of volume-limited HSBGs $(25.9 \%)$. Therefore, although we could lose some gas-rich LSBGs and HSBGs when selecting volume-limited samples, this would not affect our results too much because we just lose a very small fraction of gas-rich galaxies, and the fraction we lose for LSBGs and HSBGs is very similar.

Furthermore, we compare the gas fraction in our volume-limited LSBGs with that of small sample of LSBGs in McGaugh \& de Blok (1997) to see whether our volume-limited LSBGs have quite different gas fraction from the previous studies or not. For example, the gas fraction in McGaugh \& de Blok (1997) for LSBGs is from $17 \%$ to $77 \%$ which is very similar to the range of our volume-limited LSBGs (15\% to $70 \%)$. And the median value of gas fraction for the LSBGs in McGaugh \& de Blok (1997) is $40.1 \%$, which is not very different from our volume-limited LSBGs (34.6\%), only 5.5\% higher than ours. This means that our volume-limited LSBGs could be a reasonable sample to show the properties of LSBGs.

\section{SUMMARIES}

In this paper, we continue our studies on the properties of a large sample of LSBGs from SDSS. We select a relatively large volume-limited complete sample of LSBGs from SDSS-DR4 following Zhong et al. (2008) to study their properties and compare their properties with HSBGs. This large sample of LSBGs is useful to study the statistical properties of LSBGs. And by using the volume-limited samples of LSBGs and HSBGs, we can avoid the bias introduced from the differences in the distributions of the redshift and absolute magnitude for our samples. Moreover our volume-limited LSBGs have similar stellar masses to HSBGs that further remove the effect of stellar mass. The results can be summarized as follows.

1. LSBGs tend to have lower effective dust attenuations in $z$ band with median value of $0.34 \mathrm{mag}$, which is 0.11 lower than that of HSBGs. This means that LSBGs contain less dust than HSBGs.

2. The distributions of stellar masses are nearly the same for the two samples, both of which are with median values of $2.19 \times 10^{10} M_{\odot}$. However, the median value of stellar metallicities, $\log \left(Z / Z_{\odot}\right)$, for LSBGs is still 0.11 dex lower than that of HSBGs. And LSBGs have higher mass-to-light ratios (in $z$ band, Kauffmann et al. 2003). The median value of mass-to-light ratios in $z$ band $\left(M_{*} / L_{z}\right)$ after extinction correction is 1.11 , which is 0.15 higher than that of HSBGs.

3. LSBGs are likely to have higher gas fraction than that of HSBGs, with the median value of gas fraction $8.7 \%$ higher. And there is a tight correlation between gas fraction and surface brightness that galaxies with lower surface brightness have higher gas fraction, and the slope of gas fraction vs. surface brightness for LSBGs is slightly steeper than that of HSBGs. Although we may lose some gas-rich LSBGs when selecting our volume-limited LSBGs, however, we find that the gas-fraction of our volume-limited LSBGs is not much different from all the parent LSBGs (34.6\% vs. 39.8\%). Moreover our large sample of LSBGs have the similar gas fraction to the McGaugh \& de Blok (1997) with a small sample of LSBGs.

4. LSBGs have lower SFRs with median value of $1.77 M_{\odot} \mathrm{yr}^{-1}$, which is $0.86 M_{\odot} \mathrm{yr}^{-1}$ lower than that of HSBGs which suggest that HSBGs underwent more recent star forming activities than 
LSBGs. However the median value of SFRs for LSBGs in our work is not as low as in previous studies. Moreover, the SFRs decrease with decreasing surface brightness, i.e. the higher the surface brightness are, the stronger present star forming activities they have.

5. The specific SFRs of LSBGs are also lower than that of HSBGs, and the specific SFRs also decrease with decreasing surface brightness, but the slope of the relation between specific SFRs and surface brightness is a little steeper than that of SFRs and surface brightness, which may be due to the effects of stellar masses that are further removed in calculating specific SFRs.

In summary, LSBGs have different star formation histories from HSBGs and HSBGs may have stronger star forming activities than LSBGs.

\section{ACKNOWLEDGEMENTS}

We are grateful to the referee for constructive comments from which this paper has benefitted. This work was supported by the National Natural Science Foundation of China (Grant Nos. 10933001, 10973006, 10973015 and 10673002); the National Basic Research Program of China (973 Program; Nos. 2007CB815404 and 2007CB815406); and the Young Researcher Grant of National Astronomical Observatories, Chinese Academy of Sciences. We thank the useful MPA/JHU catalogs.

Funding for the SDSS and SDSS-II has been provided by the Alfred P. Sloan Foundation, the Participating Institutions, the National Science Foundation, the U.S. Department of Energy, the National Aeronautics and Space Administration, the Japanese Monbukagakusho, the Max Planck Society, and the Higher Education Funding Council for England. The SDSS Web site is http://www.sdss.org/ The SDSS is managed by the Astrophysical Research Consortium for the Participating Institutions. The Participating Institutions are the American Museum of Natural History, Astrophysical Institute Potsdam, University of Basel, University of Cambridge, Case Western Reserve University, University of Chicago, Drexel University, Fermilab, the Institute for Advanced Study, the Japan Participation Group, Johns Hopkins University, the Joint Institute for Nuclear Astrophysics, the Kavli Institute for Particle Astrophysics and Cosmology, the Korean Scientist Group, the Chinese Academy of Sciences (LAMOST), Los Alamos National Laboratory, the Max-Planck-Institute for Astronomy (MPIA), the Max-Planck-Institute for Astrophysics (MPA), New Mexico State University, Ohio State University, University of Pittsburgh, University of Portsmouth, Princeton University, the United States Naval Observatory, and the University of Washington.

\section{References}

Adelman-McCarthy, J., et al. 2006, ApJS, 162, 38

Alard, C. 2011, ApJ, 728, L47

Bergvall, N., Zackrisson, E., Caldwell, B. 2010, MNRAS, 405, 2697

Blanton M. R., et al. AJ, 129, 2562

Brinchmann, J., Charlot, S., White, S. D. M., Tremonti, C., Kauffmann, G., Heckman, T., Brinkmann, J. 2004, MNRAS, 351, 1151

Boissier, S., et al. 2008, ApJ, 681, 244

Boissier, S., Prantzos, N. 2000, MNRAS, 312, 398

Burkholder, V., Impey, C., \& Sprayberry, D. 2001, AJ, 122, 2318

Dalcanton, J. J., Spergel, D. N., Summers, F. J. 1997, ApJ, 482, 659

Freeman, K. C. 1970, ApJ, 160, 811

Galaz, G., Herrera-Camus, R., Garcia-Lambas, D, Padilla, N. 2011, ApJ, 728, 74

Galaz, G., Villalobos, A., Infante, L., Donzelli, C. AJ, 2006, 131, 2035

Gallazzi, A., Charlot, S., Brinchmann, J., White, S. D. M., Tremonti, C. A. 2005, MNRAS, 362, 41

Gerritsen, J. P. E., de Blok, W. J. G. 1999, A\&A, 342, 655 
Haberzettl, L., Bomans, D. J., Dettmar, R. J., Pohlen, M. 2007a, A\&A, 465, 95

Haberzettl, L., Bomans, D. J., Dettmar, R. J. 2007b, A\&A, 471, 787

Hinz, J. L., Rieke, M. J., Rieke, G. H., Willmer, C. N. A., Misselt, K., Engelbracht, C. W., Blaylock, M. 2007, ApJ, 663,895

Impey, C. D., Burkholder, V., Sprayberry, D. 2001, AJ, 122, 2341

Impey, C. D., Sprayberry, D., Irwin, M. J., Bothun, G. D. 1996, ApJS, 105, 209

Kauffmann, G., et al. 2003, MNRAS, 341, 54

Kennicutt, R. C. 1989, ApJ, 344, 685

Kennicutt, R. C., Tamblyn, P., Congdon, C. W. 1994, ApJ, 435, 22

Liang, Y. C., et al. 2010, MNRAS, 409, 213

McGaugh, S. 1994, ApJ, 426, 135

McGaugh, S., Bothun, G., Schombert, J. 1995, AJ, 110, 573

McGaugh, S., \& de Blok, W. J. G. 1997, ApJ, 481, 689

Mei, L., Yuan, W. M., Dong, X. B. 2009, RAA, 9, 269

Minchin, R. F., et al. 2004, MNRAS, 355, 1303

Mo, H. J., McGaugh, S., Bothun, G. D. 1994, MNRAS, 267, 129

Monnier Ragaigne, D., van Driel, W., O’Neil, K., Schneider, S. E., Balkowski, C., Jarrett, T. H. 2003a, A\&A, 408, 67

Monnier Ragaigne, D., van Driel, W., Schneider, S. E., Balkowski, C., Jarrett, T. H. 2003b, A\&A, 408, 465

Morelli, L., Corsini, E. M., Pizzella, A., Bonta, E. D., Coccato, L., Mendez-Abreu J., Cesetti, M. 2012, MNRAS, in press.

O’Neil, K., Bothun, G. 2000, AJ, 119, 136

O’Neil, K., Bothun, G., Cornell, M. E. 1997, AJ, 113, 1212

Pizzella, A., Corsini, E. M., Sarzi, M., Magorrian, J., Mendez-Abreu J., Coccato, L., Morelli, L., Bertola, F. 2008, MNRAS, 387, 1099

Rosenbaum, S. D., Bomans, D. J. 2004, A\&A, 422, L5

Rosenbaum, S. D., Krusch, E., Bomans, D. J., Dettmar, R. J. 2009, A\&A, 504, 807

Schombert, J., Maciel, T., McGaugh, S. S. Advances in Astronomy/Hindawi, 2011, in press

Schombert, J., McGaugh, S. S., Eder, J A. AJ, 2001, 121, 2320

Sprayberry, D., Bernstein, G. M., Impey, C. D. 1995, ApJ, 438, 72

Stoughton, C., et al. 2002, AJ, 123, 485

Strauss, M. A., et al. 2002, AJ, 124, 1810

Trachternach, C., Bomans D. J., Haberzettl L., Dettmar, R. J. 2006, A\&A, 458, 341

Ulmer, M. P., Adami, C., Durret, F., Ilbert, O., Guennou, L. 2011, A\&A., 528, A36

van den Hoek, L. B., de Blok, W. J. G., van der Hulst, J. M., de Jong, T. 2000, A\&A, 357, 397

van der Hulst, J. M., Skillman, E. D., Smith, T. R., Bothun, G. D., McGaugh, S. S., de Blok, W. J. G. 1993, AJ, 106, 548

York, D. J., et al. 2000, AJ, 120, 1579

Zhang, W., Li, C., Kauffmann, G., Zou, H., Catinella, B., Shen, S., Guo, Q., \& Chang, R. X. 2009, MNRAS, 397 , 1243

Zhong, G. H., Liang, Y. C., Liu, F. S., Hammer, F., Hu, J. Y., Chen, X. Y., Deng, L. C., Zhang, B. 2008, MNRAS, 391,986

Zhong, G. H., Liang, Y. C., Hammer, F., Chen, X. Y., Deng, L. C., Flores, H. 2010, A\&A, 520, A69

This paper was prepared with the RAA IATEX macro v1.2. 